\title{
MiR-129-5p is required for histone deacetylase inhibitor-induced cell death in thyroid cancer cells
}

Patrick Brest ${ }^{1,2}$, Sandra Lassalle 1,2,3,4, Veronique Hofman ${ }^{1,2,3,4}$ Olivier Bordone '3, Virginie Gavric Tanga ${ }^{3}$, Christelle Bonnetaud ${ }^{3}$, Chimene Moreilhon ${ }^{2,5,6}$, Geraldine Rios, ${ }^{2,6}$, José Santini, ,7,8, Pascal Barbry2,6, Catharina Svanborg, ${ }^{9}$, Baharia Mograbi $^{1,2}$, Bernard Mari, ${ }^{2,6}$ and Paul Hofman ${ }^{1,2,3,4}$

\footnotetext{
${ }^{1}$ INSERM ERI-21, EA4319, Nice, France

${ }^{2}$ University of Nice Sophia-Antipolis, Nice, France

${ }^{3}$ Centre Hospitalier Universitaire de Nice, Hôpital Pasteur, Tumorothèque, Centre de Ressource Biologique INSERM, Nice, France

${ }^{4}$ Centre Hospitalier Universitaire de Nice, Hôpital Pasteur, Laboratoire de Pathologie Clinique et Expérimentale, Nice, France

${ }^{5}$ Centre Hospitalier Universitaire de Nice, Hôpital Pasteur, Laboratoire d'oncohématologie, Nice, France

${ }^{6}$ CNRS UMR 6097, Institut de Pharmacologie Moléculaire et Cellulaire, Valbonne, France

${ }^{7}$ Département d'otorhinolaryngologie, Centre Hospitalier Universitaire de Nice, Hôpital Pasteur, Nice, France

${ }^{8}$ Centre Antoine-Lacassagne, Nice, France

${ }^{9}$ Department of Microbiology, Immunology, and Glycobiology, Institute of Laboratory Medicine, Lund University, Lund, Sweden
}

(Correspondence should be addressed to P Hofman at IRCAN, Institute of Research on Cancer and Ageing of Nice, Université de Nice Sophia-Antipolis, Avenue de Valombrose, 06107 Nice, France; Email: hofman@unice.fr)

(P Breast, S Lassalle, V Hofman, B Mograbi and P Hofman are now at IRCAN, Institute of Research on Cancer and Ageing of Nice, INSERM, CNRS, Nice, France)

\begin{abstract}
The molecular mechanism responsible for the antitumor activity of histone deacetylase inhibitors (HDACi) remains elusive. As HDACi have been described to alter miRNA expression, the aim of this study was to characterize HDACi-induced miRNAs and to determine their functional importance in the induction of cell death alone or in combination with other cancer drugs. Two $\mathrm{HDACi}$, trichostatin A and vorinostat, induced miR-129-5p overexpression, histone acetylation and cell death in BCPAP, TPC-1, 8505C, and CAL62 cell lines and in primary cultures of papillary thyroid cancer (PTC) cells. In addition, miR-129-5p alone was sufficient to induce cell death and knockdown experiments showed that expression of this miRNA was required for HDACi-induced cell death. Moreover, miR-129-5p accentuated the anti-proliferative effects of other cancer drugs such as etoposide or human $\alpha$-lactalbumin made lethal for tumor cells (HAMLET). Taken together, our data show that miR-129-5p is involved in the antitumor activity of $\mathrm{HDACi}$ and highlight a miRNA-driven cell death mechanism.
\end{abstract}

Endocrine-Related Cancer (2011) 18 711-719

\section{Introduction}

Mutation and epigenetic reorganization of chromatin are key events of tumor initiation and progression that lead to alteration of gene expression (Hanahan \& Weinberg 2000, Jones \& Baylin 2007). These epigenetic events include hypermethylation of promoters, modification of histone tails, and aberrant miRNA expression. Furthermore, histone deacetylases are overexpressed in tumors and promote carcinogenesis by blocking the transcription of tumor suppressor genes (Bolden et al. 2006). Finally, because of their efficacy in treatment of human malignancies many histone deacetylase inhibitors (HDACi) are currently being tested in clinical trials (Bolden et al. 2006, Prince et al. 2009), and have shown biological effects including tumor cell growth arrest, differentiation, and apoptosis. In thyroid cancer, several studies have reported that HDACi induce cell death through 
caspase activation and B-cell CLL/lymphoma 2 (BCL2) downregulation (Greenberg et al. 2001, Catalano et al. 2005, Mitsiades et al. 2005). Moreover, HDACi were shown to sensitize tumor cells to chemotherapy, radiation, and surgery (Catalano et al. 2006, Noguchi et al. 2009). However, the molecular mechanisms responsible for the antitumor activity of HDACi remain elusive.

miRNAs are non-protein-coding small RNAs that negatively regulate the expression of hundreds of mRNA by inhibiting their translation or by promoting their degradation. miRNA has been identified as important developmental regulators and their misexpression may play a critical role in tumorigenesis and in clinical outcome (Calin \& Croce 2006a,b, Esquela-Kerscher \& Slack 2006). Moreover, previous studies have shown that cancer drugs including HDACi quickly modulate expression of several miRNAs (Scott et al. 2006), including miR-129-5p in bladder carcinoma cell lines (Dyrskjot et al. 2009). The precise impact of changes in miRNA expression in response to HDACi in cancer drug-associated tumor regression remains essentially unknown.

Few treatment options are currently available for poorly differentiated thyroid carcinomas and the prognosis is poor. Therefore, we have assessed the efficacy of HDACi on a panel of thyroid cancer cells ranging from well-differentiated carcinomas to aggressive, poorly differentiated tumors. We show that HDACi-induced cell death correlated with a specific increase in miR-129-5p expression, regardless of the oncogene involved and that miR-129-5p is necessary for HDACi-induced cell death. Taken together, the results suggest that miR-129-5p might be used as a biomarker for in vitro HDACi treatment efficacy.

\section{Materials and methods}

\section{Reagents and HDACi}

Etoposide, staurosporine and the most widely used HDACi trichostatin A (TSA) were provided by SigmaAldrich and the clinically relevant HDACi vorinostat (SAHA) by Cayman Chemical (Ann Arbor, MI, USA). PremiR and antagomiR were purchased from Applied Biosystems (Life Technologies, Carlsbad, CA, USA) (PM10195, AM10195, AM17110, and AM17010). Human $\alpha$-lactalbumin made lethal for tumor cells (HAMLET) was produced from native purified human milk $\alpha$-lactalbumin on an oleic acid-conditioned ion exchange matrix as described previously (Svensson et al. 2000).

\section{Cell culture}

The culture conditions for TPC-1, BCPAP, and 8505C (kind gift of Pr Santoro, Napoli, Italy) and CAL62 (kind gift of the Centre Antoine-Lacassagne (CAL), Nice, France) cells have been described previously (Gioanni et al. 1991, Ito et al. 1993, Fabien et al. 1994) and were grown in DMEM with 5\% FCS, sodium pyruvate, and penicillin/streptomycin (Life Technologies). The toxicity of HDACi treatment on primary cultures of thyrocytes derived from thyroid tissue of adenomas or hyperplasias or cancer cells derived from thyroid tissue of papillary thyroid carcinoma, obtained immediately after thyroidectomy, was tested. Patient consent was obtained and the institutional review board approved the project. After dissociation, primary culture cells were maintained in culture medium complemented with TSH. Contamination by fibroblast was controlled by the pathologists. An antibody against vimentin (Ventana, Paris, France; diluted 1:200; $45 \mathrm{~min}$ ) was used to detect a potential contamination by fibroblast. No immunostaining was observed. Conversely, all cultured cells were immunostained by antibodies against HBME1 (Ventana; diluted 1:100; $45 \mathrm{~min}$ ) and/ or CK19 (Ventana; diluted 1:200; $45 \mathrm{~min}$ ).

For all experiments, cells were grown to $70 \%$ confluence, and TSA (330 $\mathrm{nM})$ or vorinostat $(2.5 \mu \mathrm{M})$, were added for $16 \mathrm{~h}$ in fresh complete medium. Control cells were left untreated. The effect of HDACi on cell viability (cell cycle, 2,3-bis(2-methoxy-4-nitro-5sulfophenyl)-2H-tetrazolium-5-carboxanilide (XTT) and morphology) was assessed by biochemical assays as well as by microarray analyses of miRNA and mRNA.

\section{miRNA transfection}

Cells were plated at 100000 cells/well in a six-well plate and transfected with synthetic premiRs or antagomiRs using Lipofectamine RNAiMAX reagent (Life Technologies), following the manufacturer's instructions at a final concentration of 5 and $20 \mathrm{nM}$ respectively.

\section{Western blotting}

Western blotting was performed as described previously (Brest et al. 2007). The antibodies used were rb-acH4; ms-phospho-ser139-H2AX or rb-poly-ADP-ribose polymerase (PARP) (06-866, 05-636, AB16661 from Millipore, Billerica, MA, USA) and rb-GAPDH-HRP conjugate (Santa Cruz Biotechnology, Inc., Santa Cruz, CA, USA). Immunoreactive bands were revealed by enhanced chemiluminescence (Amersham-GE Healthcare, Velizy-Villacoublay, France) using Kodak film. 


\section{Isolation of RNA}

Isolation of RNA from cultured cells or human samples was performed as described previously (Triboulet et al. 2007, Pottier et al. 2009).

\section{miRNA microarrays}

miRNAs of BCPAP cells treated with TSA $(330 \mathrm{nM}$, $16 \mathrm{~h}$ ) were profiled by the University of Nice SophiaAntipolis Core facility (www.microarray.fr) using home-made arrays (Triboulet et al. 2007, Pottier et al. 2009) and deposited in NCBI Gene Expression Omnibus (GEO; http://www.ncbi.nlm.nih.gov/geo/) under series GSE19934 and platform GPL4717. Briefly, total RNA was labeled with $\mathrm{Cy} 3$ or Cy5 fluorescent dyes (a dye-swap method was used) using the Ulysis Alexa fluor nucleic acid labeling kit (Amersham Bioscience), and miRNA was then isolated using the mirVana miRNA isolation kit (Applied Biosystems). Three independent experiments were performed to identify miRNA up- or downregulated in cells. For each oligonucleotide microarray, TIF images containing data from each fluorescence channel were quantified with GenePix Pro 6.1 program (Molecular Devices, Sunnyvale, CA, USA). Normalization was obtained with limmaGUI Software from Bioconductor (www.bioconductor.org) (Wettenhall \& Smyth 2004, Ritchie et al. 2007), according to the variance stabilization and calibration for microarray data (VSN) approach described by Huber et al. (2002).

\section{Expression microarrays}

miR-129-5p transfected TPC-1 cells were profiled using pangenomic microarrays printed with the human RNG/MRC oligonucleotide collection as described previously (Le Brigand et al. 2006) and deposited in GEO under series GSE19933 and platform GPL3241. Total RNA ( $2 \mu \mathrm{g}$ ) was amplified using the Amino Allyl MessageAmp aRNA kit (Ambion) according to the manufacturer's instructions. Cy3- and Cy5-labeled aRNA were hybridized on the array for $17 \mathrm{~h}$ at $62{ }^{\circ} \mathrm{C}$ (a dye-swap method was used). Arrays were scanned and quantified as described earlier.

Intra- and inter-slide normalization of three independent experiments was performed by the Global Loess and the quantile methods respectively. Means of ratios from all comparisons were calculated and a $B$ test analysis was performed by the Limma package available from Bioconductor (Gentleman et al. 2004). Differentially expressed genes were selected using a Benjamini-Hochberg correction of the $P$ value for multiple tests, with a positive $B$ value. Microarray data was archived in GEO under reference GSEX. Before analysis, the Sylamer algorithm was used to characterize the specificity of miR-129-5p on global mRNA gene expression. The human targets of the
A

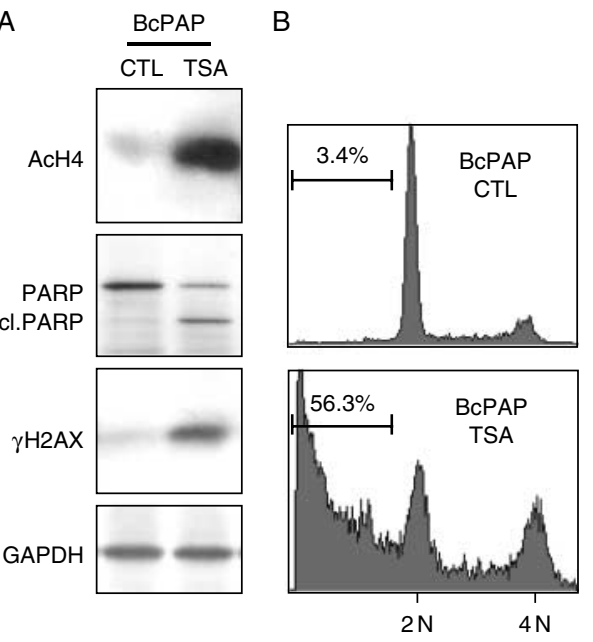

C

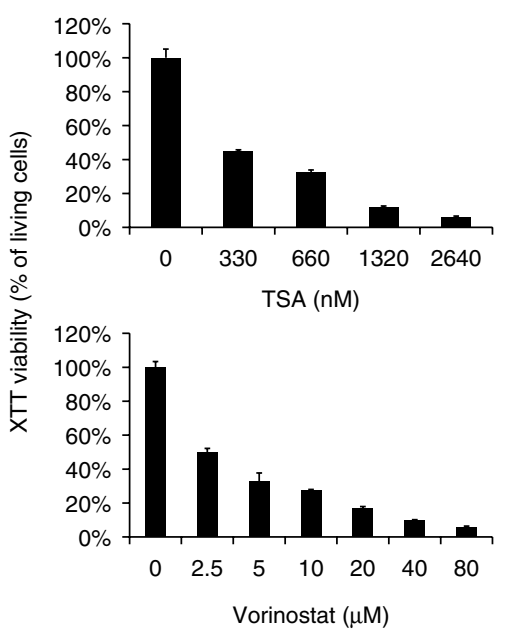

D

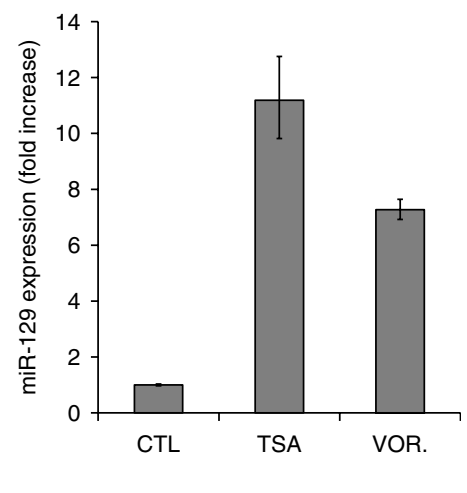

Figure $1 \mathrm{HDACi}$-induced cell death of BCPAP thyroid cells. (A) TSA induces: histone $\mathrm{H} 4$ acetylation, poly (ADP-ribose) polymerase (PARP) cleavage and histone H2AX phosphorylation. Proteins of BCPAP thyroid cells treated without (CTL) or with TSA (18 h, $330 \mathrm{nM} / \mathrm{l})$ were analyzed by western blotting. (B) DNA quantification of the subG1 population by cytometry of BCPAP cells treated without (CTL) or with TSA (C) BCPAP cells treated with either TSA (upper panel) or vorinostat (lower panel) showed concentrationdependent induced cytotoxicity as determined by the XTT viability assay (histogram). (D) The expression of miRNA in BCPAP cells treated without or with TSA $(18 \mathrm{~h}, 330 \mathrm{nM} / \mathrm{l})$ or vorinostat $(2.5 \mu \mathrm{M} / \mathrm{l})$ was analyzed by RT-PCR. Results represent the fold increase in miR-129-5p in treated cells vs untreated cells used as a reference. 
Table 1 Microarray results of statistically regulated microRNA in BCPAP cells treated with trichostatin A $(330 \mathrm{nmol} / \mathrm{l} ; n=3$ experiments)

\begin{tabular}{lccc}
\hline Name & LogFC & $\boldsymbol{P}$ value & Adj. $\boldsymbol{P}$ value \\
\hline Mir-513 (hsa) & 0.95 & $1.20 \times 10^{-5}$ & $1.66 \times 10^{-2}$ \\
Mir-663 (hsa) & 0.97 & $2.55 \times 10^{-5}$ & $1.96 \times 10^{-2}$ \\
Mir-129 (hsa.xtr) & 0.73 & $1.01 \times 10^{-3}$ & $8.05 \times 10^{-2}$ \\
Mir-373 (hsa) & 0.94 & $1.10 \times 10^{-3}$ & $8.06 \times 10^{-2}$ \\
\hline
\end{tabular}

LogFC, logarithm of fold-change.

differentially expressed miRNAs were predicted using public web-based prediction tools, such as PicTar, TargetScan, and miRBase Targets.

\section{Quantitative PCR}

Quantitative PCR (QPCR; sequences described in the Supplementary methods, see section on supplementary data given at the end of this article) for mRNA was carried out using the SYBR Green Master Mix from Applied Biosystems. QPCR for miRNAs was carried out using probes for miR-129-5p and RNU19-RNA, and the TaqMan miRNA kit from Applied Biosystems.

\section{Viability assay}

Cell survival was examined using the Cell Proliferation Kit II (XTT) from Roche and quantification of the DNA fragmented-subG1 population was performed by cell cycle analysis as described previously (Brest et al. 2007). All experiments were repeated at least three times, and each XTT experimental sample (5000 cells/ well) was repeated at least in quadruplicate wells for each experiment. The data are average values \pm s.D. of representative experiments.

\section{Statistical analysis}

For statistical analysis the SPSS16 program was used (SPSS, Chicago, IL, USA). The results were evaluated for statistical significance by the Student's $t$-test or the ANOVA test. Error bars represent the S.D. of the mean. $P$ values $<0.05$ were regarded as significant.

\section{Results}

\section{HDACi increase miR-129-5p expression in thyroid cancer cells}

The molecular mechanisms responsible for the antitumor activity of HDACi remain poorly characterized and the relationship between the sensitivity to HDACi and miRNAs expression has not been investigated in thyroid tumor cells. To test this, we first treated the BCPAP papillary thyroid cancer cell line with the HDACi-reference TSA (330 nmol/l). The BCPAP cell line was sensitive to TSA (Fig. 1A), as shown by an increase in histone $\mathrm{H} 4$ acetylation. TSA-induced apoptosis, as shown by PARP cleavage (Fig. 1A), an elevated fraction of cells in subG1 (Fig. 1B), and phosphorylation of histone H2AX (Fig. 1A) that forms before the appearance of internucleosomal DNA fragments and the externalization of phosphatidylserine to the outer membrane leaflet (Rogakou et al. 2000). The decrease in viability was also observed after XTT quantification (Fig. 1C), the concentrations selected for the rest of the study were $330 \mathrm{nmol} / 1$ and $2.5 \mu \mathrm{mol} / 1$ for TSA and vorinostat respectively. Thus, RNA from the BCPAP cell line treated or not with TSA overnight $(330 \mathrm{nmol} / \mathrm{l})$ was analyzed using a miRNA microarray (Triboulet et al. 2007, Pottier et al. 2009). TSA-treated cells showed increased expression of several miRNAs, such as miR-129, miR-513, and miR-663 (Table 1). Specific TaqMan PCR (Fig. 1D) confirmed that TSA or vorinostat treatment induced an increase in miR-129-5p (mature form) expression in comparison

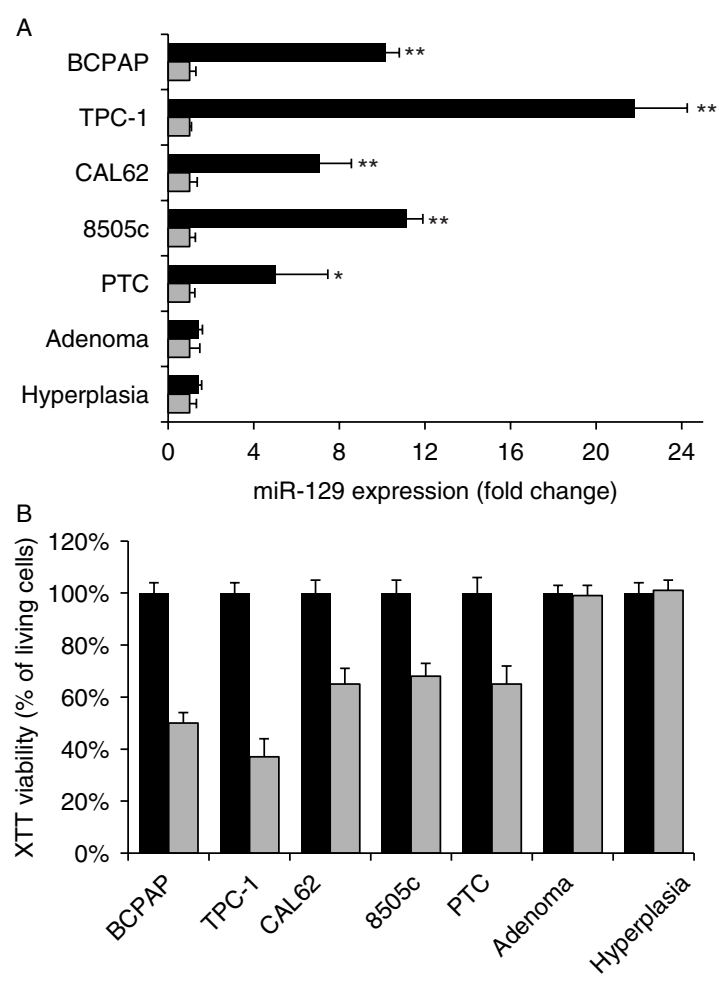

Figure 2 HDACi increases cell death and mir-129-5p expression. Malignant thyroid cells (TPC-1, BCPAP, 8505c, CAL62, and primary papillary thyroid cancer PTC $n=3$ cells) and non-malignant primary culture cells (adenoma $n=3$ and hyperplasia $n=4)$ were treated without or with TSA (18 h, $330 \mathrm{nmol} / \mathrm{l})$. (A) The expression of miRNA was analyzed by RT-PCR. Results represent the fold increase in miR-129-5p in treated cells vs untreated cells used as a reference. (B) HDACiinduced cytotoxicity analyzed by XTT. * $P<0.05$; ${ }^{\star *}, P<0.01$. 
with untreated cells whereas other changes in miRNA expression were not confirmed by this method.

We corroborate these results obtained for BCPAP (BRAF V600E) with another papillary thyroid cell line TPC-1 (RET/PTC1) and the anaplastic thyroid cell lines 8505c (BRAF V600E) and CAL62 (KRAS G ${ }^{12}$ R) when treated with the HDACi-reference TSA (330 nmol/l). Specific TaqMan PCR confirmed that TSA treatment induced an increase in miR-129-5p (mature form) expression ranging from 7- (CAL62) to 21-fold (TPC-1) in comparison with untreated cells (Fig. 2A). The highest induction was found in papillary cell lines (BCPAP and TPC-1), which correlated with their high sensitivity to cell death (Fig. 2B). These results indicate that TSA induces apoptotic cell death in thyroid cancer cells, regardless of the cancer type and their oncogenic status.

Moreover, the increased cell death and expression of miR-129-5p was only observed in primary papillary thyroid cancer (PTC) treated with TSA while no increase was observed in primary thyroid cell cultures form adenomas or hyperplasias treated with TSA (Fig. 2). The results indicate that $\mathrm{HDACi}$ treatment upregulates miR-129-5p expression in tumor cells, but not in non-malignant cells.

\section{miR-129-5p is cytotoxic for thyroid cancer cells}

We next examined the effect of miR-129-5p overexpression on cell death. For this purpose we used TPC-1 cells that we showed to be the most sensitive and found that miR-129-5p-transfection is cytotoxic for cancer cells, as shown by increased propidium iodide staining, loss of viability (XTT), and PARP cleavage (Fig. 3A-C respectively). This result was confirmed in all the tested cell lines (Fig. 3D). Moreover, no or few cell death was observed in thyroid primary cells (data not shown) showing a specific effect for transformed cancer cells. We then analyzed the influence of limited miR-129-5p overexpression (always $<25$-fold increase) on transcript levels using human pan genomic arrays, as described previously (Triboulet et al. 2007, Pottier et al. 2009). Data collected from three independent biological

B

C
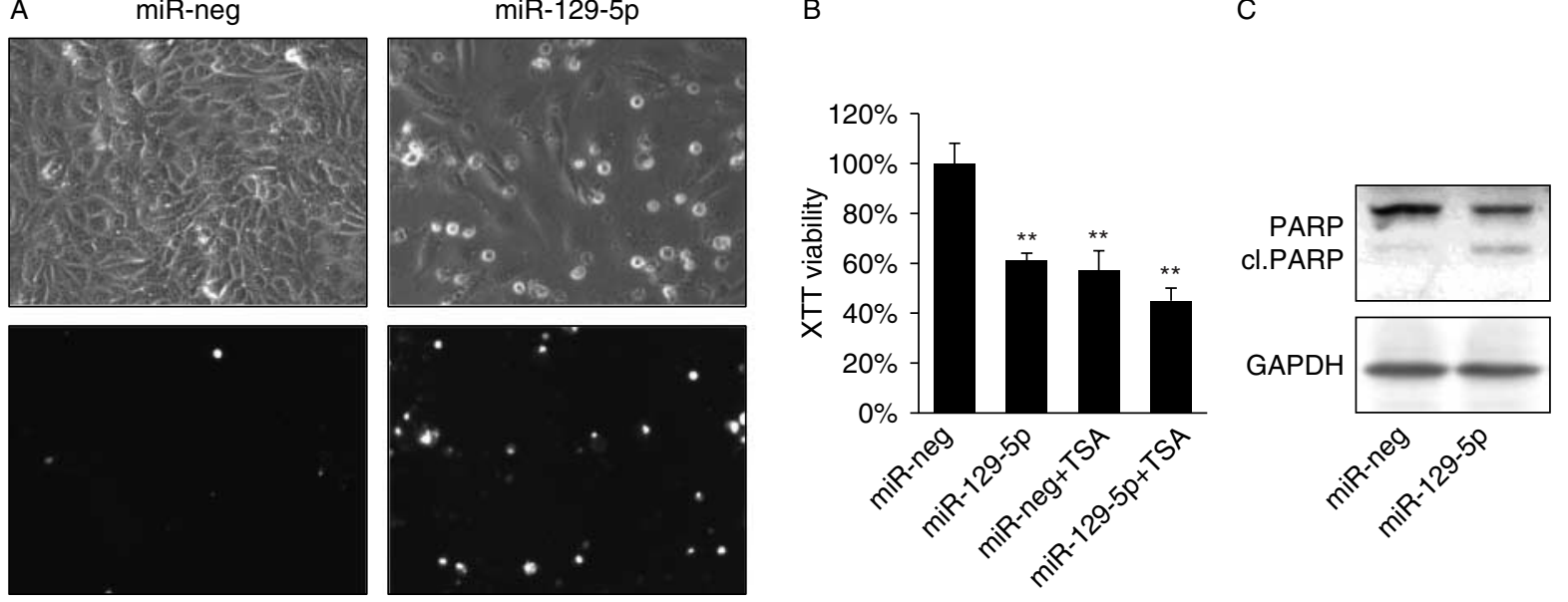

$\mathrm{D}$

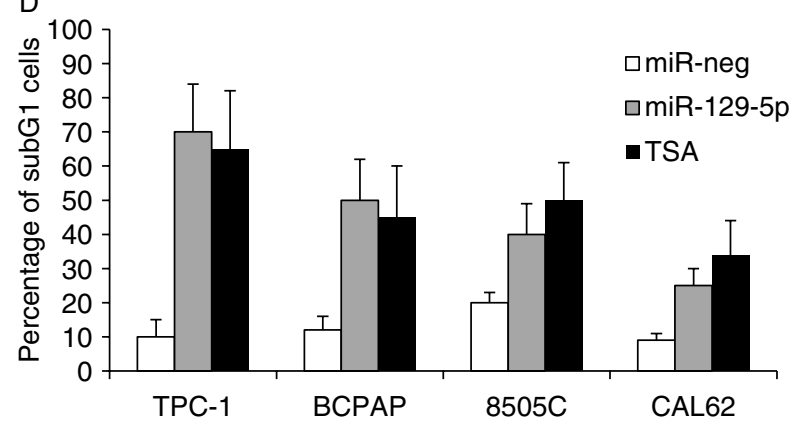

Figure 3 miR-129-5p is sufficient for induction of cell death. TPC-1 cells were transfected with either control miRNA (miRNA-neg) or miR-129-5p. MiR-129-5p transfected TPC-1 cells showed induced cytotoxicity as determined by contrast phase microscopy (upper pictures), propidium iodide staining $(5 \mu \mathrm{g} / \mathrm{ml})$ of late apoptotic cells (lower pictures; A), by XTT viability assay (B), and by PARP cleavage by western blotting $(C)$. Moreover, these results were confirmed in all the thyroid cell lines by SubG1 quantification after cell cycle analysis by cytometry (D). ${ }^{\star *}, P<0.01$ 
experiments on TPC- 1 cells, revealed that a total of 224 transcripts were significantly modulated (102 up- and 122 downregulated, $P<0.01$ ) following premiR-129$5 \mathrm{p}$ transfection compared with the control condition (Supplementary Table 1, see section on supplementary data given at the end of this article). Analysis of this signature with Ingenuity Pathway Software (Redwood City, CA, USA) indicated a significant enrichment for 'molecular functions' with terms such as 'cellular compromise, cellular growth and proliferation, cell cycle, or cell death' (Supplementary Table 2, see section on supplementary data given at the end of this article). Using our bioinformatic tool 'MicroToptable' (Pottier et al. 2009), we then looked for potential overrepresentation of miR-129-5p-predicted targets among the downregulated transcripts (cut off equal to 8.0 for the $\log _{2}$ (signal), to -1 for the $\log _{2}$ (ratio), and to 0.01 for the adjusted $P$ values) and isolated 42 transcripts corresponding to putative targets (Supplementary Table 3, see section on supplementary data given at the end of this article). By RT-PCR, we validated a decrease in UDP- $N$-acetyl- $\alpha$-D-galactosamine: polypeptide $N$-acetylgalactosaminyltransferase 1 (GALNT1; $2^{-2.0} \sim-4$ fold), a gene previously described to be regulated by miR-129-5p (Dyrskjot et al.2009). Interestingly, we also confirmed an increase in the pro-apoptotic protein programed cell death 2 $\left(2^{1.7} \sim 3.2\right.$ fold; Supplementary Table 3 , see section on supplementary data given at the end of this article) concomitant to a decrease in its repressor BCL6 $\left(-2^{-2.4} \sim-5.3\right.$ fold $)$. These results were in accord with the predictive action of this miR-129-5p on BCL6 and GALNT1 using the PicTar and TargetScan algorithm. These results suggest that induction of miR-129-5p expression may participate in HDACiinduced cell death possibly through the repression of GALNT1 and BCL6.

\section{miR-129-5p is necessary for HDACi-induced cell death}

To examine if the induction of miR-129-5p expression was required for $\mathrm{HDACi}$-induced cell death, TPC-1 cells were transfected for $24 \mathrm{~h}$ with specific antagomiR directed against miR-129-5p and then incubated in the presence of TSA (330 nmol/l, $24 \mathrm{~h}$; Fig. 4A). AntagomiR-129-5p significantly blocked TSA-increased cell death in contrast to antagomiR-neg transfected cells
A

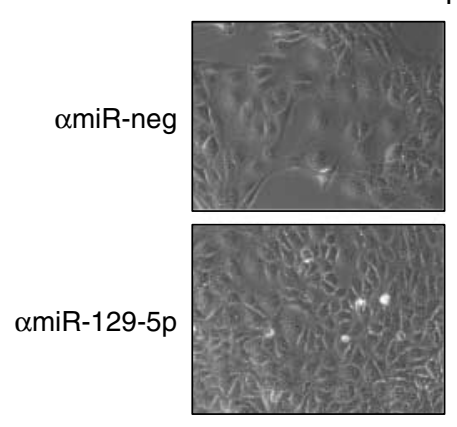

B

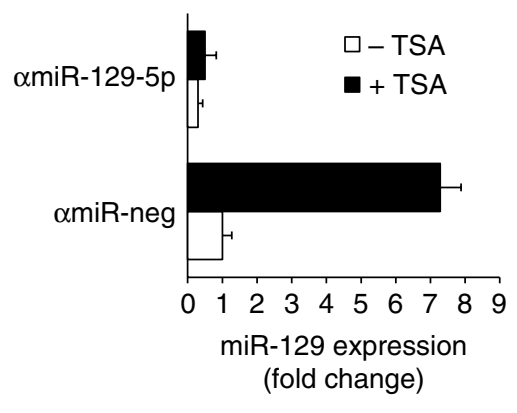

- TSA
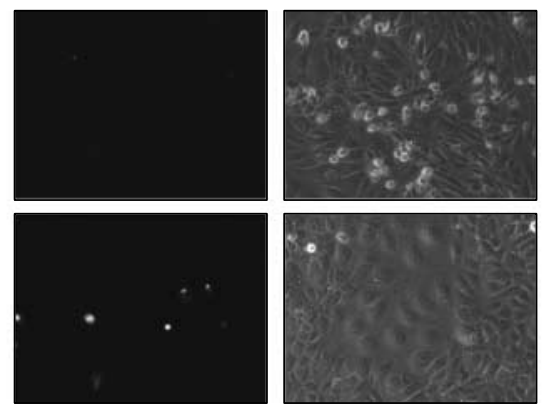

TSA
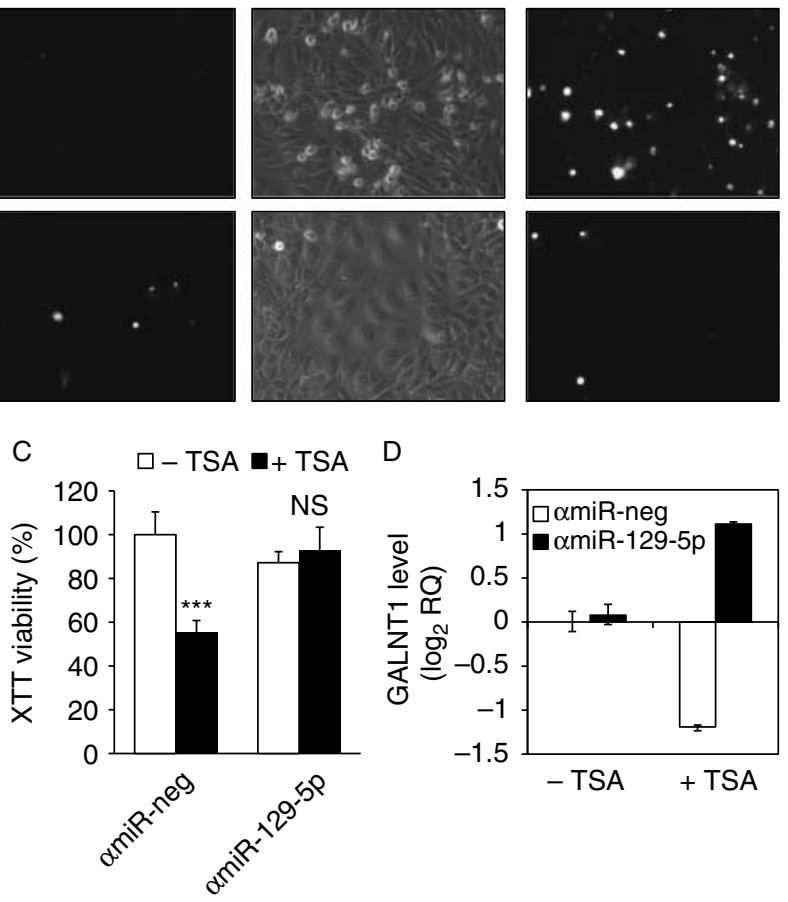

Figure 4 miR-129-5p is necessary for HDACi-induced cell death. (A) Phase contrast microscopy and propidium iodide staining of late apoptotic cells of cells transfected with antagomiR-neg ( $\alpha$ miR-neg) or antagomiR-129-5p ( $\alpha$ miR-129-5p) and incubated without $(-)$ or with (+) TSA. (B) RT-PCR of miR-129-5p was performed to confirm the effect of antagomiR. (C) TPC-1 cells were transfected with either antagomiR-neg or antagomiR-129-5p for $48 \mathrm{~h}$ and incubated without or with TSA (330 nmol/l) for the last $24 \mathrm{~h}$. Cell death was followed using an XTT viability assay. (D) GALNT1 expression was quantified in cells after transfection of antagomiR and incubated without or with TSA $(330 \mathrm{nmol} / \mathrm{l})$ for the last $24 \mathrm{~h}$. ${ }^{\star \star *}, P<0.001$. 
treated with HDACi, as shown by both microscopy and the XTT viability assay (Fig. 4A-C). Furthermore, HDACi-dependent repression of GALNT1 expression was reverted by antagomiR-129-5p. GALNT1 was even overexpressed in presence of the antagomiR and TSA (Fig. 4D). However, HDACi-dependent BCL6 decreased expression was not reversed by antagomiR-129-5p showing a potential combined effect of TSA-induced microRNA on BCL6 expression (data not shown). In conclusion, silencing of miR-129-5p in thyroid cells was able to decrease HDACi-induced cell death, demonstrating that miR-129-5p expression is a key mediator of HDACi-mediated killing of tumor cells.

\section{miR-129-5p promotes drug-induced cell death}

HDACi have previously been shown to induce cell death in response to other cancer drugs such as etoposide, cisplatin, or HAMLET (Brest et al. 2007). To examine if miR-129-5p may have a similar effect, we transfected TPC-1 cells with either miR-129-5p or miR-CTL for $24 \mathrm{~h}$ before the addition of increasing concentrations of etoposide or HAMLET. Combination with miR-129-5p was additive with HAMLET, staurosporine, or etoposide treatment (Fig. 5). The loss of viability in response to HAMLET $(0.3 \mathrm{mg} / \mathrm{ml}, 24 \mathrm{~h})$ was $40 \%$ in $\mathrm{miR}-\mathrm{CTL}$ transfected control cells, but increased to $70 \%$ in tumor cells transfected with miR-129-5p. A similar increase in cell death was obtained in cells treated with staurosporine or etoposide. In CAL62 cells, the results were similar to

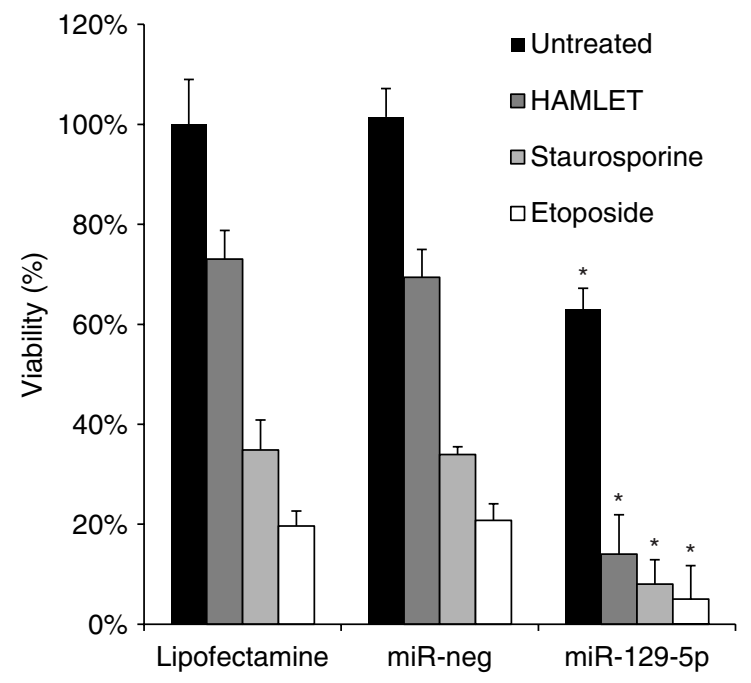

Figure 5 miR-129-5p promotes drug-induced cell death. TPC-1 cells were transfected for $24 \mathrm{~h}$ with either control miRNA (miRNA-neg) or miR-129-5p (lipofectamine alone was used as control) and incubated with HAMLET $(0.3 \mathrm{mg} / \mathrm{ml})$ or etoposide $(0.3 \mathrm{mM})$ or staurosporine $(0.3 \mu \mathrm{M})$ for $24 \mathrm{~h}$. Cell death was followed using a XTT viability assay. ${ }^{*}, P<0.05$.
TPC-1 cells (data not shown). Thus, while miR-129-5p affects tumor cell viability per se, it also accentuates the effect to different cancer drugs.

\section{Discussion}

Thyroid cancers represent a broad spectrum of neoplastic disorders that include differentiated cancers with good prognosis after surgery and radioiodine therapy, but also poorly differentiated carcinomas and some medullary carcinomas that are associated with poor prognosis. These tumors are often inoperable, resistant to radiotherapy and have a high tendency to metastasize. Conventional chemotherapy is often ineffective, highlighting the need for novel therapeutic strategies. Despite the therapeutic efficacy of HDACi in several malignancies, the mechanism of action remains unclear. In this study, we show that the activity of miR-129-5p is directly related to the efficiency of HDACi as well as to substances with a related or synergistic mechanism of action. For this study, we used the most widely used HDACi TSA for which the development as a therapeutic agent has been neglected partly due to: i) the high cost of production and isolation from fermentation broth and ii) the clinically relevant HDACi vorinostat has been approved by the U.S. FDA for the treatment of cutaneous T-cell lymphoma and has been tested for treatment of patients with metastatic and/or locally advanced or locally recurrent thyroid cancer (clinical trial phase II).

Few studies have evaluated the effect of HDACi on thyroid cancer cells and no study has compared the sensitivity of HDACi on a panel of thyroid cancers harboring different oncogenes. It has been shown that vorinostat induces cell death in FRO81-2 anaplastic thyroid cancer cells (BRAF V600E) by activation of caspase and degradation of Bcl-2 family proteins. (Mitsiades et al. 2005). However, to our knowledge, no study has investigated the effect of HDACi on the overexpression of specific miRNA in thyroid cancer cells. We show here that both TSA and vorinostat have a potent effect on miR-129-5p expression in thyroid cancer cells, while cultures of non-malignant primary cells were insensitive to these drugs. In addition, miR129-5p was strongly induced in the entire thyroid cell lines tested (Fig. 2) and in some other cell lines such as A549 (lung carcinoma) and MEL-501 (melanoma) cells (data not shown). While this work was in progress, it was shown that the HDACi 4-phenylbutyric acid restores the expression of miR-129-2 (miR-129-5p precursor) in colorectal cancers (Bandres et al. 2009), which is in agreement with our results.

We next investigated the impact of miR-129-5p expression on HDACi-induced cell death. Overexpression 
of miR-129-5p alone was able to induce cell death in our model of TPC-1 thyroid cancer, confirming a recent study showing that miR-129-5p was cytotoxic in bladder carcinoma cell lines (Dyrskjot et al. 2009). A direct link between miR-129-5p and the two putative targets GALNT1 and SOX4 was documented (Dyrskjot et al. 2009). In our model, transfection with miR-129-5p, led to a strong decrease in GALNT1 (approximately fourfold; Supplementary Table 3 , see section on supplementary data given at the end of this article) and BCL6 ( 5.2-fold) expression, but to a modest decrease in SOX4 $(\sim 1.6$-fold, data not shown) expression. Using our bioinformatic tools, we also isolated, for further functional studies, a group of 42 transcripts (including GALNT1) corresponding to putative miR-129-5p targets (Supplementary Table 1, see section on supplementary data given at the end of this article). We showed that miR-129-5p knockdown blocked HDACi-induced cell death in our model. This result showed that miR-129-5p is both sufficient and mandatory for HDACi-induced cell death and obviously through a GALNT1-dependent function.

Over the last decade, the explosion of efforts into drug discovery has led to the development of a large group of HDACi, many of which have been shown preclinically to have potent antitumor activity. Moreover, HDACi have been shown to synergize with many anti-cancer agents, including cytotoxic agents such as paclitaxel (Dowdy et al. 2006), cisplatin (Strait et al. 2005), etoposide and doxorubicin (Marchion et al. 2004), HAMLET (Brest et al. 2007), or radiotherapy (Noguchi et al. 2009). In this study, we showed that miR-129-5p enhanced the antitumor effects of staurosporine, etoposide, and HAMLET when used in combination (Fig. 5).

There is a need to better define and target HDACi for clinical use in specific patient groups. Specific biomarkers for different tumor types or mechanisms may be vital to stratify patients and tumors into subgroups that are responsive, as well as to enable target modulation to be monitored. Our findings provide new evidence for a miRNA-driven antitumor effect of HDACi and therefore we propose that miR-129-5p might be useful as a biomarker in following the HDACi-induced response in tumor cells, to define susceptible and resistant tumors.

\section{Supplementary data}

This is linked to the online version of the paper at http://dx. doi.org/10.1530/ERC-10-0257.

\section{Declaration of interest}

The authors declare that there is no conflict of interest that could be perceived as prejudicing the impartiality of the research reported.

\section{Funding}

This work was supported by the Institut National du Cancer (INCa, France; grants PL0079, 07/3D1616/Pdoc-110-32/NGNC, and R06080AA, 2006, 2007, 2008), the French Medical Research (FRM, France; 2005, 2006), the European Community (MICROENVIMET, FP7-HEALTH-F2-2008-201279, 2008) and the CHUN-CNRS, the Gunnar Nilsson Cancer Foundation (2006), the Sharon D Lund Foundation, the Swedish Cancer Society, the Swedish Research Council, the Medical Faculty, Lund University, the Söderberg, Pediatric Cancer, the Anna-Lisa and Sven-Erik Lundgren and the Knut and Alice Wallenberg Foundations, the Lund City Jubileumsfond, the John and Augusta Persson Foundation for Medical Research, the Maggie Stephens Foundation, the Inga-Britt and Arne Lundberg Foundation, the H J Forssman Foundation for Medical Research, and the Royal Physiographic Society.

\section{Acknowledgements}

We acknowledge the excellent support of the Nice SophiaAntipolis Functional Genomics Platform at which the microarray experiments were carried out and Peter Storm for help preparing this manuscript.

\section{References}

Bandres E, Agirre X, Bitarte N, Ramirez N, Zarate R, Roman-Gomez J, Prosper F \& Garcia-Foncillas J 2009 Epigenetic regulation of microRNA expression in colorectal cancer. International Journal of Cancer $\mathbf{1 2 5}$ 2737-2743. (doi:10.1002/ijc.24638)

Bolden JE, Peart MJ \& Johnstone RW 2006 Anticancer activities of histone deacetylase inhibitors. Nature Reviews. Drug Discovery 5 769-784. (doi:10.1038/nrd2133)

Brest P, Gustafsson M, Mossberg AK, Gustafsson L, Duringer C, Hamiche A \& Svanborg C 2007 Histone deacetylase inhibitors promote the tumoricidal effect of HAMLET. Nature Reviews. Drug Discovery 67 11327-11334. (doi:10.1158/0008-5472.CAN-07-1153)

Calin GA \& Croce CM $2006 a$ MicroRNA signatures in human cancers. Nature Reviews. Cancer 6 857-866. (doi:10.1038/nrc1997)

Calin GA \& Croce CM 2006b MicroRNAs and chromosomal abnormalities in cancer cells. Oncogene 25 6202-6210. (doi:10.1038/sj.onc.1209910)

Catalano MG, Fortunati N, Pugliese M, Costantino L, Poli R, Bosco O \& Boccuzzi G 2005 Valproic acid induces apoptosis and cell cycle arrest in poorly differentiated thyroid cancer cells. Journal of Clinical Endocrinology and Metabolism 90 1383-1389. (doi:10.1210/jc.2004-1355)

Catalano MG, Fortunati N, Pugliese M, Poli R, Bosco O, Mastrocola R, Aragno M \& Boccuzzi G 2006 Valproic acid, a histone deacetylase inhibitor, enhances sensitivity to doxorubicin in anaplastic thyroid cancer cells. Journal of Endocrinology 191 465-472. (doi:10.1677/joe.1.06970)

Dowdy SC, Jiang S, Zhou XC, Hou X, Jin F, Podratz KC \& Jiang SW 2006 Histone deacetylase inhibitors and 
paclitaxel cause synergistic effects on apoptosis and microtubule stabilization in papillary serous endometrial cancer cells. Molecular Cancer Therapeutics 5 2767-2776. (doi:10.1158/1535-7163.MCT-06-0209)

Dyrskjot L, Ostenfeld MS, Bramsen JB, Silahtaroglu AN, Lamy P, Ramanathan R, Fristrup N, Jensen JL, Andersen CL, Zieger K et al. 2009 Genomic profiling of microRNAs in bladder cancer: miR-129 is associated with poor outcome and promotes cell death in vitro. Cancer Research 69 4851-4860. (doi:10.1158/0008-5472.CAN-08-4043)

Esquela-Kerscher A \& Slack FJ 2006 Oncomirs - microRNAs with a role in cancer. Nature Reviews. Cancer 6 259-269. (doi:10.1038/nrc1840)

Fabien N, Fusco A, Santoro M, Barbier Y, Dubois PM \& Paulin C 1994 Description of a human papillary thyroid carcinoma cell line. Morphologic study and expression of tumoral markers. Cancer 73 2206-2212. (doi:10.1002/ 1097-0142(19940415)73:8<2206::AIDCNCR2820730828> 3.0.CO;2-M)

Gentleman RC, Carey VJ, Bates DM, Bolstad B, Dettling M, Dudoit S, Ellis B, Gautier L, Ge Y, Gentry J et al. 2004 Bioconductor: open software development for computational biology and bioinformatics. Genome Biology 5 R80. (doi:10.1186/gb-2004-5-10-r80)

Gioanni J, Zanghellini E, Mazeau C, Zhang D, Courdi A, Farges M, Lambert JC, Duplay H \& Schneider M 1991 Characterization of a human cell line from an anaplastic carcinoma of the thyroid gland. Bulletin du Cancer $\mathbf{7 8}$ 1053-1062.

Greenberg VL, Williams JM, Cogswell JP, Mendenhall M \& Zimmer SG 2001 Histone deacetylase inhibitors promote apoptosis and differential cell cycle arrest in anaplastic thyroid cancer cells. Thyroid 11 315-325. (doi:10.1089/ 10507250152039046)

Hanahan D \& Weinberg RA 2000 The hallmarks of cancer. Cell 100 57-70. (doi:10.1016/S0092-8674(00)81683-9)

Huber W, von Heydebreck A, Sultmann H, Poustka A \& Vingron M 2002 Variance stabilization applied to microarray data calibration and to the quantification of differential expression. Bioinformatics 18 (Suppl 1) S96-S104. (doi:10.1093/bioinformatics/18.suppl_1.S96)

Ito T, Seyama T, Iwamoto KS, Hayashi T, Mizuno T, Tsuyama N, Dohi K, Nakamura N \& Akiyama M 1993 In vitro irradiation is able to cause RET oncogene rearrangement. Cancer Research 53 2940-2943.

Jones PA \& Baylin SB 2007 The epigenomics of cancer. Cell 128 683-692. (doi:10.1016/j.cell.2007.01.029)

Le Brigand K, Russell R, Moreilhon C, Rouillard JM, Jost B, Amiot F, Magnone V, Bole-Feysot C, Rostagno P, Virolle V et al. 2006 An open-access long oligonucleotide microarray resource for analysis of the human and mouse transcriptomes. Nucleic Acids Research 34 e87. (doi:10.1093/nar/gkl485)

Marchion DC, Bicaku E, Daud AI, Richon V, Sullivan DM \& Munster PN 2004 Sequence-specific potentiation of topoisomerase II inhibitors by the histone deacetylase inhibitor suberoylanilide hydroxamic acid. Journal of Cellular Biochemistry 92 223-237. (doi:10.1002/jcb.20045)
Mitsiades CS, Poulaki V, McMullan C, Negri J, Fanourakis G, Goudopoulou A, Richon VM, Marks PA \& Mitsiades N 2005 Novel histone deacetylase inhibitors in the treatment of thyroid cancer. Clinical Cancer Research 11 3958-3965. (doi:10.1158/1078-0432.CCR-03-0776)

Noguchi H, Yamashita H, Murakami T, Hirai K, Noguchi Y, Maruta J, Yokoi T \& Noguchi S 2009 Successful treatment of anaplastic thyroid carcinoma with a combination of oral valproic acid, chemotherapy, radiation and surgery. Endocrine Journal 56 245-249. (doi:10.1507/endocrj.K08E-016)

Pottier N, Maurin T, Chevalier B, Puissegur MP, Lebrigand K, Robbe-Sermesant K, Bertero T, Lino Cardenas CL, Courcot E, Rios G et al. 2009 Identification of keratinocyte growth factor as a target of microRNA-155 in lung fibroblasts: implication in epithelial-mesenchymal interactions. PLoS ONE 4 e6718. (doi:10.1371/journal.pone.0006718)

Prince HM, Bishton MJ \& Harrison SJ 2009 Clinical studies of histone deacetylase inhibitors. Clinical Cancer Research 15 3958-3969. (doi:10.1158/1078-0432.CCR-08-2785)

Ritchie ME, Silver J, Oshlack A, Holmes M, Diyagama D, Holloway A \& Smyth GK 2007 A comparison of background correction methods for two-colour microarrays. Bioinformatics 23 2700-2707. (doi:10.1093/ bioinformatics/btm412)

Rogakou EP, Nieves-Neira W, Boon C, Pommier Y \& Bonner WM 2000 Initiation of DNA fragmentation during apoptosis induces phosphorylation of $\mathrm{H} 2 \mathrm{AX}$ histone at serine 139. Journal of Biological Chemistry 275 9390-9395. (doi:10.1074/jbc.275.13.9390)

Scott GK, Mattie MD, Berger CE, Benz SC \& Benz CC 2006 Rapid alteration of microRNA levels by histone deacetylase inhibition. Cancer Research 66 1277-1281. (doi:10.1158/0008-5472.CAN-05-3632)

Strait KA, Warnick CT, Ford CD, Dabbas B, Hammond EH \& Ilstrup SJ 2005 Histone deacetylase inhibitors induce G2checkpoint arrest and apoptosis in cisplatinum-resistant ovarian cancer cells associated with overexpression of the Bcl-2-related protein Bad. Molecular Cancer Therapeutics 4 603-611. (doi:10.1158/1535-7163.MCT-04-0107)

Svensson M, Hakansson A, Mossberg AK, Linse S \& Svanborg C 2000 Conversion of alpha-lactalbumin to a protein inducing apoptosis. PNAS 97 4221-4226. (doi:10. 1073/pnas.97.8.4221)

Triboulet R, Mari B, Lin YL, Chable-Bessia C, Bennasser Y, Lebrigand K, Cardinaud B, Maurin T, Barbry P, Baillat V et al. 2007 Suppression of microRNA-silencing pathway by HIV-1 during virus replication. Science $\mathbf{3 1 5}$ 1579-1582. (doi:10.1126/science.1136319)

Wettenhall JM \& Smyth GK 2004 limmaGUI: a graphical user interface for linear modeling of microarray data. Bioinformatics 20 3705-3706. (doi:10.1093/bioinformatics/bth449)

Received in final form 6 September 2011 Accepted 26 September 2011 Made available online as an Accepted Preprint 26 September 2011 